\title{
MILIEUX A LA CHLOROMYCÉTINE, POUR L'ISOLEMENT DES DERMATOPHYTES ET DES CHAMPIGNONS LEVURIFORMES
}

\author{
Par J. COUDERT ET M. MURAT
}

L'isolement des Dermatophytes et des Champignons pathogènes, à partir des prélèvements cutanés, et plus encore à partir des selles, du pus ou de l'expectoration, est entravé par la pullulation des germes microbiens associés, saprophytes ou pathogènes. Leur développement plus rapide et plus abondant crée une concurrence susceptible de retarder ou de supplanter les germes mycosiques, de développement plus lent. Une identification correcte requiert d'abord une souche pure.

Divers procédés ont été préconisés pour entraver le développement de cette flore microbienne. L'emploi d'antiseptiques à doses très faibles: violet de gentiane, quinanil, tellurite de potassium, sulfate de cuivre, activine, ne protège pas toujours, aux doses utilisables, d'un développement microbien encore gênant, certains germes s'en accommodant, alors que le développement d'espèces intéressantes peut se trouver entravé, ou ne se fait que pauvrement et lentement.

L'acidité était déjà une caractéristique intéressante des milieux classiques de Sabouraud et de Raulin, obtenue par leur seule composition. Divers auteurs ont proposé de la renforcer par addition d'acides chlorhydrique ou citrique. L'action antimicrobienne se montre encore insuffisante, et tous les Champignons pathogènes ne s'en accommodent pas aisément.

Plus intéressante apparait l'utilisation des antibiotiques. La pénicilline a été proposée en 1950 par Vanbreuseghem, pour l'isolement des Dermatophytes. L'addition extemporanée dans chaque tube, à 10 cc. de milieu de Sabouraud, liquifié au bain-marie, de 0,5 cc. de solution stérile de pénicilline G, titrant 10.000 U.O. par cc., réalise une concentration de 500 U.O. environ par cc. de milieu. Nous avons largement utilisé ce milieu et constaté, que non seulement le déve-

Ann. de Parastrologie, T. XXX, $\mathrm{N}^{\circ} 3 .-1955$. 
loppement des bactéries associées est grandement diminué, mais de plus le développement des colonies fongiques est plus précoce, plus abondant, et la fréquence des colonies isolées sur ce milieu apparait beaucoup plus grande que sur les milieux classiques.

A l'usage, cependant, quelques inconvénients apparaissent : la labilité de la pénicilline en solution oblige à une préparation extemporanée et ne permet qu'une conservation de quelques jours à basse température. D'autre part, la fréquence actuelle des germes pénicillino-résistants dans les produits pathologiques ne permet pas toujours à l'action bactério-statique de s'exercer suffisamment, pour protéger la vitalité du Champignon associé. Dans le but de remédier à ces inconvénients, nous utilisons de préférence la chloromycétine. Deux types de milieux s'avèrent intéressants pour le travail courant de la pratique clinique.

Milieu de Sabouraud à la chloromycétine. - Nous utilisons le milieu de Sabouraud modifié par Langeron :

\begin{tabular}{|c|c|}
\hline & \\
\hline e ou maltose $\ldots \ldots \ldots \ldots \ldots \ldots$ & $20 \mathrm{gr}$. \\
\hline Gélose .. & \\
\hline
\end{tabular}

Le milieu, filtré ou décantẻ à chaud, est réparti en flacons et stérilisé à l'autoclave. Encore liquide ou liquéfié au bain-marie, il est additionné de la solution de chloromycétine dans l'eau, stérilisée par simple ébullition. La dose employée est de $1 \mathrm{cc}$. de solution titrant $10.000 \gamma$ par cc. (soit une capsule de $0,25 \mathrm{gr}$. dans $25 \mathrm{cc}$. d'eau) pour un flacon de $30 \mathrm{cc}$., ou encore de $30 \mathrm{cc}$. pour un ballon de un litre. La concentration réalisée est de $330 \gamma$ par cc. On mélange ; puis le milieu peut être, soit stocké, soit réparti en tubes.

Extrait de malt gélosé à la chloromycétine. - Milieu spécialement adapté à l'isolement des levures à partir des selles, expectoration, etc. :

Extrait de malt desséché ........... $40 \mathrm{gr}$.

Gélose ....................... $20 \mathrm{gr}$.

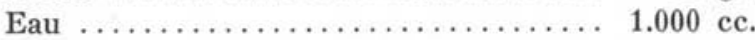

Le milieu, filtré ou décanté à chaud, est réparti en flacons et additionné de la solution de chloromycétine dans les mêmes conditions et aux mêmes doses que le milieu précédent.

La conservation de ces milieux, sans précautions spéciales, est excellente, même au bout de plusieurs mois.

Dans la pratique, le pouvoir sélectif de ces milieux est apparu beaucoup plus important que nous ne l'avions espéré, vis-à-vis des bactéries, et, dans certains cas, nous avons pu obtenir d'emblée des 
colonies pures, précocement développées et présentant leur morphologie typique. La présence de chloromycétine au taux employé est sans action sur le développement et sur la morphologie des espèces pathogènes. D'autre part, il semble bien que l'absence de concurrence microbienne est l'élément essentiel permettant à la colonie de développer rapidement ses caractères spécifiques et ses fructifications caractéristiques. Ce gain de temps est très appréciable pour le clinicien, trop souvent rebuté par la lenteur du diagnostic mycologique, par rapport aux diagnostics bactériologiques courants. Le diagnostic des espèces courantes en est grandement facilité, et, dans le cas des Levures, auxquelles convient particulièrement le milieu à l'extrait de malt, le gain de temps réalisé dans la purification de la souche est des plus intéressants. Dans ce cas, où l'absence complète de germes microbiens est indispensable, avant de passer à l'identification proprement dite, il nous est arrivé de pouvoir passer directement du milieu à la chloromycétine aux épreuves de fermentation. Dans les cas où quelques germes persisteraient encore, un seul passage sur liquide de Raulin suffit à terminer la purification.

Dans le cas des Dermatophytes, l'isolement des agents des teignes suppurées s'avère beaucoup plus facile et fréquent. Les échecs sont absolument exceptionnels, même si l'on ne peut ensemencer qu'un nombre restreint de tubes. Dans les cas douteux de mycoses surinfectées, cette sensibilité apparait d'autant plus remarquable.

Pour les teignes sèches, l'avantage peut paraître moins évident. Néanmoins, dans certains cas douteux, du fait de l'irritation due à des traitements intempestifs, l'élimination des germes saprophytes permet un développement plus rapide et plus fréquent du germe pathogène subsistant encore en faible proportion.

Il nous semble done que ces deux types de milieux d'isolement auraient avantage à être largement utilisés comme milieux de routine, pour l'isolement des Champignons pathogènes, par les Laboratoires d'analyses biologiques.

[Travail du Laboratoire de Parasitologie ( $\mathrm{P}^{*}$ Ch. GarIN) et de la Clinique Dermatologique de l'Antiquaille ( $\mathrm{P}^{\mathrm{r}} \mathrm{J}$. GATÉ, Lyon)] 